\title{
O burnout em profissionais de enfermagem que atuam em um complexo psiquiátrico
}

The burnout in nursing professionals who work in a psychiatric complex

Burnout en profesionales de enfermería que trabajan en un complejo psiquiátrico

\section{Ana Rebeca Soares de Medeiros ${ }^{\mathrm{I}}$, Carla Braz Evangelista ${ }^{\mathrm{II}}$, Ronny Anderson de Oliveira Cruz ${ }^{\mathrm{III}}$ Ericka Holmes Amorim ${ }^{\mathrm{IV}}$, José Madson Medeiros Souza ${ }^{\mathrm{V}}$}

Resumo: Objetivo: investigar o burnout em profissionais de enfermagem que atuam em um Complexo Psiquiátrico. Método: pesquisa exploratória, quantitativa, realizada com 42 profissionais de enfermagem. Para a coleta, utilizou-se um instrumento constando de dados sociodemográficos e profissionais, e o Cuestionario para la Evaluación del Síndrome de Quemarse por el Trabajo - CESQT. Resultados: quando considerado o percentil, 17 $(40,5 \%)$ profissionais de enfermagem encontravam-se com níveis muito baixos sugestivos de burnout. O tempo de trabalho na instituição teve associação com o desgaste psíquico $(\mathrm{p}=0,03)$. Conclusão: em média, os entrevistados não apresentaram valores sugestivos do burnout, porém o tempo de atuação esteve associado às dimensões do agravo. Ressalta-se que medidas de prevenção devem ser realizadas para eliminar ou reduzir as possíveis chances de surgimento do agravo.

Descritores: Saúde mental; Saúde do trabalhador; Esgotamento profissional

\begin{abstract}
Aim: To investigate burnout in nursing professionals working in a Psychiatric Complex. Method: exploratory, quantitative research conducted with 42 nursing professionals. For the collection, we used an instrument consisting of sociodemographic and professional data, and the Cuestionario para la Evaluación del Síndrome de Quemarse por el Trabajo - CESQT. Results: when considering the percentile, 17 (40.5\%) nursing professionals had very low levels suggestive of burnout. Working time in the institution was associated with psychic burnout $(p=0.03)$. Conclusion: on average, the respondents did not show values suggestive of burnout, but
\end{abstract}

IEnfermeira. Graduada pelo Centro Universitário de João Pessoa - UNIPÊ. João Pessoa, Paraíba, Brasil. E-mail: becasmedeiros@hotmail.com ORCID ID: https://orcid.org/0000-0001-7519-8152

${ }^{I I}$ Enfermeira. Mestre em Enfermagem pelo Programa de Pós-Graduação em Enfermagem da Universidade Federal da Paraíba - UFPB. Professora Assistente do Curso de Enfermagem do Centro Universitário de João Pessoa - UNIPÊ. João Pessoa, Paraíba, Brasil. E-mail: carlabrazevangelista@gmail.com ORCID ID: https://orcid.org/0000-0001-7063-1439

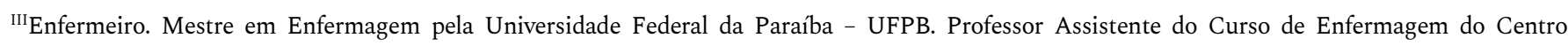
Universitário de João Pessoa - UNIPÊ. João Pessoa, Paraíba, Brasil. E-mail: ronnyufpb@gmail.com ORCID ID: https://orcid.org/0000-0001-6443-7779

${ }^{I V}$ Enfermeira. Mestre em Modelos de Decisão e Saúde - UFPB. Professora Assistente do Curso de Enfermagem do Centro Universitário de João Pessoa - UNIPÊ. João Pessoa, Paraíba, Brasil. E-mail: ericka.amorim@unipe.br ORCID ID: https://orcid.org/0000-0003-2763-3652

${ }^{\vee}$ Enfermeiro e Engenheiro de produção. Mestre em Gestão e Economia da Saúde pela Universidade Federal de Pernambuco - UFPE. Professor Assistente e Coordenador Adjunto do Curso de Enfermagem do Centro Universitário de João Pessoa - UNIPÊ. João Pessoa, Paraíba, Brasil. Email: madson.medeiros@unipe.edu.br ORCID ID: https://orcid.org/0000-0002-4447-1870 
the length of action was associated with the dimensions of the injury. It is noteworthy that preventive measures should be performed to eliminate or reduce the possible chances of emergence of the disease.

Descriptors: Mental health; Occupational Health; Burnout, Professional

Resumen: Objetivo: investigar el agotamiento en los profesionales de enfermería que trabajan en un complejo psiquiátrico. Método: investigación exploratoria cuantitativa realizada con 42 profesionales de enfermería. Para la recopilación, utilizamos un instrumento que consta de datos sociodemográficos y profesionales, y la Cuestionário para la Evaluación del Síndrome de Quemarse por el Trabajo - CESQT. Resultados: al considerar el percentil, 17 (40.5\%) profesionales de enfermería tenían niveles muy bajos que sugerían agotamiento. El tiempo de trabajo en la institución se asoció con agotamiento psíquico $(\mathrm{p}=0.03)$. Conclusión: en promedio, los encuestados no mostraron valores sugestivos de agotamiento, pero la duración de la acción se asoció con las dimensiones de la lesión. Es de destacar que se deben tomar medidas preventivas para eliminar o reducir las posibles posibilidades de aparición de la enfermedad.

Descriptores: Salud mental; Salud laboral; Agotamiento Professional

\section{Introdução}

Atualmente, há uma certa preocupação com a saúde do empregado, em decorrência da relação com a produtividade. Para alcançar as metas impostas pelo serviço, é preciso ter funcionários saudáveis e competentes, entretanto, em muitas empresas, o empregado trabalha sob pressão, o que acarreta patologias, insatisfações e desmotivações que podem levá-lo a apresentar fadiga, distúrbios do sono, estresse e a Síndrome de Burnout. ${ }^{1}$

A Síndrome de Burnout, também conhecida com Síndrome do Esgotamento Profissional, refere-se a uma resposta física e mental do trabalhador devido a uma prolongada exposição ao estresse no ambiente laboral. ${ }^{2}$ É um tipo de estresse ocupacional, em que o trabalhador se sente consumido física e emocionalmente, o que culmina em exaustão, agressividade e irritação. ${ }^{3}$

É crescente a preocupação referente ao assunto estresse entre profissionais de saúde, uma vez que, em decorrência do desgaste laboral e do convívio com os pacientes, há o desenvolvimento de reações agudas e/ou crônicas, as quais podem desencadear a Síndrome de Burnout. ${ }^{4}$ Os enfermeiros por atuarem diretamente com os doentes, com o adoecer, a morte e os cuidados de enfermagem, os quais exigem constante atenção, estão mais predispostos a desenvolver o agravo. ${ }^{4}$ 
3 | Medeiros ARS, Evangelista CB, Cruz RAO, Amorim EH, Souza JMM

No que diz respeito aos profissionais que atuam na saúde mental, destaca-se que o trabalho exercido, por envolver em seu cotidiano problemas psíquicos, pode ocasionar sobrecarga de cunho emocional. O envolvimento destes com os pacientes que possuem transtornos psíquicos e com outros colegas de trabalho que possuem diferentes opiniões favorece a geração de sofrimento, o que interfere na qualidade do serviço prestado e contribui para o aparecimento de agravos a esse trabalhador, incluindo o acometimento pelo burnout. ${ }^{5}$

O hospital psiquiátrico possui características que favorecem a sobrecarga do profissional. ${ }^{6}$ Neste ambiente, o profissional de enfermagem precisa estar em estado permanente de alerta em decorrência das alterações de cunho psicológico do paciente (humor, sono, personalidade, agressividade e imprevisibilidade) o que pode acarretar em danos a sua saúde. ${ }^{7}$

Estudo realizado com profissionais de enfermagem de um hospital psiquiátrico demonstrou que a organização e as condições de trabalho contribuíram para o adoecimento do profissional. A repetitividade de tarefas, condições de risco, mobiliários e estrutura física inadequada e exposição a ruídos foram os fatores que contribuíram para o risco grave de adoecimento do trabalhador. ${ }^{7}$ Tal fato traz à tona a necessidade de investigações que avaliem a presença de agravos físicos, psíquicos e emocionais em profissionais de enfermagem que atuam na área de saúde mental, mais especificamente, em hospitais psiquiátricos.

Ante ao exposto, apresentou-se a seguinte questão de pesquisa: os profissionais de enfermagem que atuam em um Complexo Psiquiátrico possuem o burnout? Existe relação entre os dados sociodemográficos e profissionais, e o burnout?

Para tanto, o presente estudo objetiva investigar o burnout em profissionais de enfermagem que atuam em um Complexo Psiquiátrico.

\section{Método}


O burnout em profissionais de enfermagem que atuam em um complexo psiquiátrico I 4

Trata-se de um estudo de campo, de natureza exploratória e abordagem quantitativa, realizado em um Complexo Psiquiátrico localizado no município de João Pessoa, Paraíba, Brasil. Os funcionários do hospital são prestadores de serviço com e sem contrato de trabalho definido, ou atuam em regime estatutário.

O universo do estudo foi constituído por 24 enfermeiros e 54 técnicos de enfermagem e a amostra se deu por conveniência, a qual foi composta por 42 profissionais de enfermagem, dos quais 23 eram enfermeiros e 19 técnicos de enfermagem, que atenderam os seguintes critérios de inclusão: estar praticando suas atividades laborais no Complexo Psiquiátrico durante a realização da coleta de dados e possuir no mínimo seis meses de atuação no serviço. Cumpre assinalar que 35 profissionais não participaram do estudo pelo fato de não se encontrarem no hospital durante o período coleta de dados, ou se recusarem a preencher os instrumentos com a justificativa de que já estavam fazendo parte de outras pesquisas. Além desses, um dos participantes trabalhava a menos de seis meses na instituição, e nesse ínterim todos foram excluídos.

A coleta de dados foi realizada no período de outubro a novembro de 2017 e contou com a utilização de dois instrumentos. O primeiro elaborado pelos autores constava de dados sociodemográficos (sexo, estado civil, renda, idade) e profissionais (tempo de atuação na instituição, tempo de atuação profissional e turno de trabalho), e o segundo sobre a Síndrome de Burnout (Cuestionario para la Evaluación del Síndrome de Quemarse por el Trabajo - CESQT), validado para uso no Brasil, $8^{8}$ e adaptado para população investigada, conforme presente em estudos, ${ }^{9-10}$ uma vez que o instrumento originalmente foi desenvolvido para aplicação em docentes, porém podendo ser utilizado em diferentes culturas. ${ }^{8}$ Ressalta-se que o instrumento em questão vem sendo utilizado com diversos profissionais ${ }^{11-12}$ e por autores que validaram o original. ${ }^{11}$ 
5 | Medeiros ARS, Evangelista CB, Cruz RAO, Amorim EH, Souza JMM

O CESQT avalia as cognições, emoções e atitudes da pessoa em seu ambiente de trabalho e é composto de 20 itens que contemplam as seguintes dimensões: ilusão (It) refere-se ao desejo de atingir metas de trabalho, o que leva a fonte de satisfação pessoal; desgaste psíquico ( $\mathrm{Dp}$ ) caracteriza-se pelo esgotamento emocional e físico; indolência (In) diz respeito à presença de atitudes negativas de indiferença e cinismo em relação aos clientes da organização como pacientes ou estudantes; e, por fim, culpa (C) caracterizada por comportamentos e atitudes negativas no trabalho. ${ }^{13}$

Pontuações abaixo de 2 na dimensão ilusão e acima ou igual a 2 nas dimensões desgaste psíquico, indolência e culpa supõem altos níveis da síndrome. O escore geral denominado de Síndrome de Quemarse por el Trabajo Total (SQT Total) foi calculado a partir da seguinte formula: 20 - It $+\mathrm{Dp}+\mathrm{In}^{13}$

Além disso, considerou-se as pontuações diretas correspondentes a escala do CESQT de acordo com os percentis (P). Os escores são classificados em muito baixo quando as pontuações são menores ou iguais ao P10; baixo quando as pontuações são menores ou iguais ao P33; médio quando pontuações são menores ou iguais ao P66; alto quando as pontuações são menores que P90 e crítico quando as pontuações são maiores ou iguais ao P90. ${ }^{13}$

Os dados coletados foram codificados e digitados na planilha Microsoft Excel 2010, em forma de banco de dados eletrônico. Em seguida, foram apresentados com frequência absoluta e relativa, média, desvio padrão e intervalo de confiança. Utilizou-se o teste de normalidade Shapiro-Wilk e foi verificado que os dados não possuíam distribuição normal. Posteriormente, foram aplicados os testes não-paramétricos de Mann-Whitney e Kruskal-Wallis, sendo adotado um nível de confiança de 5\% e significância de 95\%. Estes procedimentos foram realizados por meio do software Statistical Package for the Social Sciences - SPSS versão 20.0 para Windows.

O projeto desta pesquisa foi submetido ao Comitê de Ética e Pesquisa do Unipê, conforme a resolução 466/12 e aprovado no dia 19 de outubro de 2017, sob o número do Parecer 2.339.045 e CAEE 70674917.0.0000.5176. 
O burnout em profissionais de enfermagem que atuam em um complexo psiquiátrico I 6

\section{Resultados}

Participaram do estudo 42 profissionais de enfermagem. Os dados sociodemográficos e de atuação profissional serão apresentados a seguir:

Tabela 1. Distribuição dos participantes em relação às características sociodemográficas e profissionais. João Pessoa - PB, $2017(\mathrm{~N}=42)$.

\begin{tabular}{|c|c|c|}
\hline VARIÁVEIS & $\mathbf{N}$ & $\%$ \\
\hline \multicolumn{3}{|l|}{ Sexo } \\
\hline Masculino & 06 & $14,3 \%$ \\
\hline Feminino & 36 & $85,7 \%$ \\
\hline \multicolumn{3}{|l|}{ Estado Civil } \\
\hline Solteiro(a) & 15 & $35,7 \%$ \\
\hline Casado(a) & 17 & $40,5 \%$ \\
\hline Separado(a) & 07 & $16,7 \%$ \\
\hline Viúvo(a) & 03 & $7,10 \%$ \\
\hline \multicolumn{3}{|l|}{ Renda } \\
\hline Não especificou & 02 & $4,80 \%$ \\
\hline Até 3 Salários & 31 & $73,8 \%$ \\
\hline 3 a 6 Salários & 09 & $21,4 \%$ \\
\hline \multicolumn{3}{|l|}{ Idade } \\
\hline 20-29 anos & 05 & $11,9 \%$ \\
\hline 30-39 anos & 07 & $16,7 \%$ \\
\hline 40-49 anos & 07 & $16,7 \%$ \\
\hline 50-59 anos & 15 & $35,7 \%$ \\
\hline 60-69 anos & 07 & $16,7 \%$ \\
\hline 70-79 anos & 01 & $2,40 \%$ \\
\hline \multicolumn{3}{|c|}{ Tempo de atuação profissional } \\
\hline 6 meses- 10 anos & 15 & $35,7 \%$ \\
\hline $11-20$ anos & 07 & $16,7 \%$ \\
\hline $21-30$ anos & 12 & $28,6 \%$ \\
\hline $31-40$ anos & 07 & $16,7 \%$ \\
\hline $41-50$ anos & 01 & $2,40 \%$ \\
\hline \multicolumn{3}{|c|}{ Tempo de atuação na instituição } \\
\hline 6 meses- 10 anos & 23 & $54,8 \%$ \\
\hline 11-20 anos & 04 & $9,50 \%$ \\
\hline $21-30$ anos & 10 & $23,8 \%$ \\
\hline $31-40$ anos & 04 & $9,50 \%$ \\
\hline $41-50$ anos & 01 & $2,40 \%$ \\
\hline \multicolumn{3}{|c|}{ Turno de trabalho } \\
\hline Diurno & 18 & $42,9 \%$ \\
\hline
\end{tabular}


7 | Medeiros ARS, Evangelista CB, Cruz RAO, Amorim EH, Souza JMM

\begin{tabular}{lll}
\hline Noturno & 06 & $14,3 \%$ \\
Diurno/Noturno & 18 & $42,9 \%$ \\
\hline
\end{tabular}

O quadro 1 mostra as médias, desvio padrão, intervalo de confiança das dimensões e do escore total do questionário CESQT.

\begin{tabular}{|l|c|c|c|}
\hline \multicolumn{1}{|c|}{ DIMENSÃO } & MÉDIA & DP & IC (95\%) \\
\hline Ilusão & 3,4098 & 0,75888 & $3,1702-3,6493$ \\
\hline Desgaste psíquico & 0,9125 & 0,94826 & $0,6092-1,2158$ \\
\hline Indolência & 0,7167 & 0,64957 & $0,5089-0,9244$ \\
\hline Culpa & 0,4857 & 0,44424 & $0,3473-0,6242$ \\
\hline SQT Total & 0,6940 & 0,61610 & $0,4943-0,8937$ \\
\hline
\end{tabular}

Quadro 1. Distribuição dos participantes em relação às Pontuações Diretas (PD) das escalas do CESQT. João Pessoa - PB, 2017. ( $N=42)$.

DP: Desvio Padrão. IC: Intervalo de Confiança. SQT Total: Síndrome de Quemarse por el Trabajo Total

O quadro 2 apresenta as estatísticas de cada um dos escores calculados levando-se em consideração o percentil, a fim de avaliar os níveis que revelam se o indivíduo apresenta ou não o burnout.

\begin{tabular}{|l|c|c|c|c|c|}
\hline DIMENSÃo & $\begin{array}{c}\mathbf{P} \leq \mathbf{1 0} \\
\mathbf{N}(\mathbf{\%})\end{array}$ & $\begin{array}{c}\mathbf{P} \mathbf{1 1 - 3 3} \\
\mathbf{N}(\mathbf{\%})\end{array}$ & $\begin{array}{c}\mathbf{P} \mathbf{3 4 - 6 6} \\
\mathbf{N}(\mathbf{\%})\end{array}$ & $\begin{array}{c}\mathbf{P} \mathbf{6 7 - 8 9} \\
\mathbf{N}(\mathbf{\%})\end{array}$ & $\begin{array}{c}\mathbf{P} \geq \mathbf{9 0} \\
\mathbf{N}(\mathbf{\%})\end{array}$ \\
\hline Ilusão & $3(7,10 \%)$ & $8(19,0 \%)$ & $9(21,4 \%)$ & $21(50,0 \%)$ & - \\
\hline Desgaste psíquico & $22(52,4 \%)$ & $5(11,9 \%)$ & $9(21,4 \%)$ & $2(4,80 \%)$ & $2(4,80 \%)$ \\
\hline Indolência & $8(19,0 \%)$ & $12(28,6 \%)$ & $13(31,0 \%)$ & $5(11,9 \%)$ & $2(4,80 \%)$ \\
\hline Culpa & $12(28,6 \%)$ & $11(26,2 \%)$ & $17(40,5 \%)$ & $2(4,80 \%)$ & - \\
\hline SQT Total & $17(40,5 \%)$ & $7(16,7 \%)$ & $12(28,6 \%)$ & $1(2,40 \%)$ & $2(4,80 \%)$ \\
\hline
\end{tabular}

Quadro 2- Distribuição dos participantes em relação ao percentil das escalas do CESQT. João Pessoa - PB, 2017 (42).

$\mathrm{P}=$ percentil. SQT Total: Síndrome de Quemarse por el Trabajo Total

A tabela 2 apresenta a relação entre as variáveis sociodemográficas e profissionais e as dimensões e o escore geral do burnout.

Tabela 2 - Estatística dos escores das dimensões e do escore geral, segundo variáveis sociodemográficas e laborais. João Pessoa - PB, 2017 (42). 
O burnout em profissionais de enfermagem que atuam em um complexo psiquiátrico 18

\begin{tabular}{|c|c|c|c|c|c|c|c|c|c|c|}
\hline \multirow[t]{2}{*}{ VARIÁVEIS } & \multicolumn{2}{|c|}{ It } & \multicolumn{2}{|c|}{$\mathrm{Dp}$} & \multicolumn{2}{|c|}{ In } & \multicolumn{2}{|c|}{$\mathrm{C}$} & \multicolumn{2}{|c|}{ SQT Total } \\
\hline & M & $\mathrm{p}$ & M & $\mathrm{p}$ & M & $\mathrm{p}$ & M & $\mathrm{p}$ & M & $\mathrm{p}$ \\
\hline \multicolumn{11}{|l|}{ Sexo } \\
\hline Masculino & 3,37 & 0,60 & 0,67 & 0,59 & 0,64 & 0,99 & 0,23 & 0,14 & 0,64 & 0,89 \\
\hline Feminino & 3,42 & & 0,95 & & 0,73 & & 0,53 & & 0,70 & \\
\hline \multicolumn{11}{|l|}{ Faixa etária } \\
\hline $20-29$ & 2,70 & 0,32 & 2,00 & 0,18 & 1,42 & 0,50 & 0,65 & 0,57 & 1,53 & 0,64 \\
\hline $30-39$ & 3,31 & & 0,96 & & 0,40 & & 0,43 & & 0,65 & \\
\hline $40-49$ & 0,31 & & 1,18 & & 0,55 & & 0,46 & & 0,63 & \\
\hline $50-59$ & 0,55 & & 0,56 & & 0,59 & & 0,43 & & 0,53 & \\
\hline $60-69$ & 3,60 & & 0,67 & & 0,90 & & 0,29 & & 0,68 & \\
\hline \multicolumn{11}{|l|}{ Estado Civil } \\
\hline Solteiro & 3,54 & 0,28 & 1,30 & 0,21 & 0,76 & 0,74 & 0,47 & 0,63 & 0,80 & 0,31 \\
\hline Casado & 3,36 & & 0,80 & & 0,74 & & 0,38 & & 0,72 & \\
\hline Separado/ & 3,32 & & 0,50 & & 0,57 & & 0,44 & & 0,59 & \\
\hline \multicolumn{11}{|l|}{ Divorciado } \\
\hline Viúvo & - & & 0,25 & & 0,33 & & 0,47 & & 0,20 & \\
\hline \multicolumn{11}{|l|}{ Renda } \\
\hline$<3$ salários & 3,54 & 0,33 & 0,93 & 0,59 & 0,80 & 0,12 & 0,49 & 0,09 & 0,72 & 0,61 \\
\hline $3-6$ & 0,32 & & 0,97 & & 0,39 & & 0,27 & & 0,70 & \\
\hline \multicolumn{11}{|c|}{ Tempo de atuação profissional } \\
\hline Até 10 & 3,17 & 0,19 & 1,16 & 0,45 & 0,74 & 0,59 & 0,47 & 0,36 & 0,88 & 0,72 \\
\hline $11-20$ & 0,39 & & 1,04 & & 0,52 & & 0,57 & & 0,53 & \\
\hline $21-30$ & 3,60 & & 0,65 & & 0,63 & & 0,30 & & 0,56 & \\
\hline Acima de 30 & 3,50 & & 0,67 & & 0,92 & & 0,40 & & 0,71 & \\
\hline \multicolumn{11}{|c|}{ Tempo de atuação na instituição } \\
\hline Até 10 & 3,30 & 0,19 & 1,07 & $\mathbf{0 , 0 3}$ & 0,69 & 0,79 & 0,50 & $\mathbf{0 , 0 7}$ & 0,80 & 0,59 \\
\hline $11-20$ & - & & 1,33 & & 0,66 & & 0,60 & & 0,62 & \\
\hline $21-30$ & 3,60 & & 0,63 & & 0,70 & & 0,22 & & 0,58 & \\
\hline Acima de 30 & - & & 0,06 & & 0,83 & & 0,50 & & 0,35 & \\
\hline \multicolumn{11}{|c|}{ Turno de trabalho } \\
\hline Diurno & 3,53 & 0,85 & 0,81 & 0,20 & 0,67 & 0,96 & 0,41 & 0,48 & 0,64 & 0,64 \\
\hline Noturno & 3,57 & & 0,54 & & 0,67 & & 0,37 & & 0,56 & \\
\hline Diurno/Noturno & 3,38 & & 1,14 & & 0,73 & & 0,46 & & 0,80 & \\
\hline
\end{tabular}

It: ilusão. Dp: desgaste psíquico. In: indolência. Cp: culpa. SQT Total: Síndrome de Quemarse por el Trabajo Total. M:Média. p: valor de $\mathrm{p}$

\section{Discussão}

Foi possível observar que os profissionais de enfermagem que atuam no hospital psiquiátrico apresentaram médias que não caracterizam o burnout, embora se encontrem diante de situações trabalhistas geradoras de sobrecarga e insatisfação devido a presença de grades e lotação das 
enfermarias, ruídos, odor do local, bem como carga horária excessiva de trabalho, vindo a favorecer o processo de adoecimento e comprometimento na qualidade dos serviços prestados. ${ }^{14-15}$

Foram encontradas médias altas para a dimensão ilusão pelo trabalho. Estudo realizado com psicólogos mostrou que a dimensão de Ilusão pelo trabalho atingiu média de 2,96. Tal fato demonstra que os indivíduos "frequentemente" acreditam atingir suas metas profissionais e que essas têm sido percebidas como uma importante fonte de realização profissional, assim como na presente pesquisa. ${ }^{16}$

No escore de Síndrome de Burnout geral, a maioria dos profissionais de enfermagem (40,5\%) se encontrava com níveis muito baixos para apresentar o burnout, entretanto $28,6 \%$ apresentaram nível médio. Os níveis de desgaste psíquicos, em sua maioria, também foram baixos (52,4\%). A maioria dos participantes apresentou nível médio para o burnout na dimensão indolência (31,0\%) e culpa $(40,5 \%)$, e alto para ilusão $(50 \%)$.

Estudo realizado com 311 docentes de três instituições de ensino superior nas áreas da saúde (medicina, enfermagem e educação física), humanas (letras e pedagogia) e exatas (engenharia e matemática) concluiu que a indolência esteve presente quando relacionada a situações de pior relacionamento com colegas e com alunos, pensamentos em mudar de profissão, menos tempo livre no final de semana, desejo de melhores condições de trabalho e pior percepção de saúde. ${ }^{17}$

Quando investigadas as médias dos homens e mulheres e a relação com o burnout, verificou-se que no geral homens e mulheres não apresentaram a síndrome, embora as mulheres possuam maiores médias para todos os escores. Percebe-se que o trabalho realizado é mais atrativo e promove uma maior fonte de satisfação pessoal ${ }^{13}$ para as mulheres quando comparadas com os homens.

Embora as mulheres apresentem maiores valores para todos os escores, verifica-se que não houve significância estatística, no que diz respeito a comparação das médias dos escores entre os sexos. Outro estudo investigou a Síndrome de Burnout em profissionais da saúde que trabalhavam em um hospital infantil e demostrou que não houve significância entre os sexos. 
O burnout em profissionais de enfermagem que atuam em um complexo psiquiátrico I 10

Entretanto, na pesquisa em questão, utilizou-se outro instrumento de avaliação do burnout, o Maslach Burnout Inventory-Human Service Survey. ${ }^{18}$

Ressalta-se que, diferente do que era de se esperar, os indivíduos que ganhavam mais de três salários mínimos apresentaram baixas médias de ilusão, embora não tenha sido identificada significância estatística. Corroborando com a presente pesquisa, estudo que teve como objetivo analisar os preditores da Síndrome de Burnout em enfermeiros, que atuam nos serviços de urgência pré-hospitalar móvel, a partir do Maslach Burnout Inventory, mostrou que a realização profissional teve média maior nos que possuíam valores iguais ou menores que cinco salários mínimos e menos elevada entre os que possuíam renda igual ou maior que dez salários mínimos. ${ }^{19}$

O tempo de atuação na instituição esteve associado ao aparecimento do burnout. Os profissionais que trabalham no hospital psiquiátrico de 11 a 20 anos apresentam maior média de desgaste psíquico $(\mathrm{p}=0,03)$. Deste modo, possuem mais risco de possuir esgotamento mental e físico relacionados ao trabalho. ${ }^{13}$ Outro estudo realizado com profissionais atuantes em um hospital universitário, a partir da utilização do Maslach Burnout Inventory-Human Service Survey, demostrou significância estatística entre as variáveis estado civil, renda, procura por profissional de saúde, desinteresse pela profissão e outro vínculo empregatício. Não havendo significância com a variável tempo de serviço, sexo, idade e estado civil. ${ }^{20}$

Neste contexto, estudo de revisão integrativa afirmou que fatores pessoais como sexo, idade e estado civil por si só não são considerados possíveis causadores do agravo. Por sua vez, a autonomia, as condições de trabalho e a disponibilidade de insumos interferem na atividade laboral, que somada a sobrecarga em decorrência das jornadas de trabalho, turnos fixos e baixos salários, podem ocasionar o adoecimento mental dos trabalhadores e, consequentemente, levar ao aparecimento do burnout. ${ }^{21}$ 
Verificou-se que os indivíduos que trabalhavam no turno da noite se sentiam mais realizados, e embora não tenha apresentado significância estatística, há vantagens em se trabalhar neste horário, principalmente relacionada à redução da rotatividade de pessoas nos setores, o que leva a uma maior adaptação, um trabalho de menor intensidade e uma maior facilidade na prestação de assistência, com consequente melhoria da qualidade dos cuidados que são ofertados. ${ }^{22}$

Estudo procurou analisar os fatores associados ao burnout, de acordo com o turno de trabalho dos profissionais de enfermagem, utilizando dois instrumentos: Maslach Burnout Inventory-Human Service Survey e o Demand-Control-Support. Evidenciou-se que os profissionais que trabalhavam no turno da noite apresentavam níveis menores da Síndrome de Burnout e estavam mais satisfeitos com o lazer. No turno diurno, a alta demanda de trabalho, o baixo apoio social e controle sobre o trabalho, a insatisfação com o sono e com os recursos financeiros, ser enfermeiro e sedentário estiveram associados às dimensões do burnout. Já no turno noturno, ter filhos, não possuir religião, insatisfação com o sono e lazer, menor tempo de trabalho na instituição, ser auxiliar ou técnico de enfermagem aumentaram as chances de possuir a Síndrome. ${ }^{23}$

Outra pesquisa que teve como objetivo avaliar o nível de esgotamento profissional entre profissionais de enfermagem atuantes na terapia intensiva, utilizando um questionário com informações sociodemográficas, profissionais, sobre atividades de lazer, fatores preditores e sintomas somáticos relacionados à Síndrome de Burnout e o Maslach Burnout Inventory, identificou em sua amostra que a chance de adquirir a Síndrome de Burnout foi significativamente maior entre os funcionários que trabalham em dois ou mais turnos $(\mathrm{OR}=$ 4,07; IC=95\% (1,13 - 14,5); $\mathrm{p}=0,031),{ }^{24}$ assim como na presente pesquisa, em que as médias mais altas foram para os indivíduos que trabalham em dois turnos. 
Os profissionais de Enfermagem que atuam em serviços de saúde mental estão sujeitos a diversos fatores que levam a sobrecarga de trabalho além dos já citados como a degradação das condições de infraestrutura dos serviços e a baixa remuneração. A Organização Mundial da Saúde (OMS) tem recomendado estudos sobre o impacto do trabalho em profissionais de saúde psiquiátrica, com vistas a otimizar processo organizativos, de aperfeiçoamento e de qualidade o que pode refletir de modo significativo tanto na saúde dos profissionais bem como na qualidade da assistência. ${ }^{25}$

\section{Conclusão}

O burnout no contexto psiquiátrico poderá apresentar-se como o produto de uma interação negativa entre o local, a equipe de trabalho e os pacientes, não como um fenômeno isolado, mas uma tríade com um risco ocupacional significativo. Dentre as variáveis investigadas, apenas o tempo de atuação na instituição esteve associado ao aparecimento do agravo e de suas dimensões.

Em média, os entrevistados não apresentaram o burnout. Entretanto, quando avaliados os percentis de cada indivíduo, evidenciou-se que parte dos entrevistados apresentaram níveis médios de desgaste psíquico, indolência e culpa. Poucos foram os profissionais que apresentaram níveis críticos e altos de desgaste psíquico, indolência e SQT total. E embora seja um número pequeno, tal fato se torna preocupante, na medida em que alguns profissionais da equipe de enfermagem que atuam na área da saúde mental estão apresentando esgotamento profissional, e isso poderá influenciar negativamente no cuidado prestado ao paciente.

Nos ambientes de psiquiatria, o paciente muitas vezes encontra-se em tratamento de surtos e pode ser agressivo. O fato de lidar com esses pacientes, acrescido da sobrecarga de trabalho, pode tornar os profissionais deste setor mais susceptíveis ao adoecimento mental, e a Síndrome de Burnout. Deste modo, momentos de descontração, lazer e relaxamento se fazem 
necessários. Além disso, práticas relacionadas ao cuidando do cuidador poderão auxiliar estes profissionais e reduzir as chances de um adoecimento físico, psíquico e emocional.

O estudo apresentou algumas limitações: a recusa de participantes em responder a pesquisa, reduzindo a amostra; o fato do instrumento ser preenchido pelo próprio participante, o leva a perda de algumas informações; a utilização de um único instrumento para avaliação do burnout; a realização de somente uma abordagem de pesquisa; a coleta restrita apenas aos profissionais de uma única instituição.

Desse modo, um levantamento nas equipes de enfermagem que atuam na área da saúde mental e em outras áreas, em nível estadual e brasileiro, se faz necessário para a confirmação das variáveis associadas ao aparecimento do burnout e a sua presença na população investigada. Estudos mistos são necessários para melhorar as investigações sobre os fatores associados ao aparecimento do burnout.

Aliada a isso, a pesquisa poderá servir como alerta aos profissionais de enfermagem que atuam na saúde mental sobre as possíveis chances de desenvolvimento do burnout contribuindo com o despertar da comunidade científica e da sociedade em geral para o conhecimento de um agravo à saúde cada vez mais prevalente entre os trabalhadores, corroborando para o surgimento de intervenções que implicarão na redução do número de profissionais acometidos.

\section{Referências}

1. Santos VC, Veloso CSA. A Síndrome de Burnout e seus efeitos no contrato de trabalho. Rev Cient Facerb. 2016;3(2):76-94.

2. Soldera LLO, Martins LG. Síndrome de Burnout: conceitos e observações para os gestores de recursos humanos. Leopoldianum [Internet]. 2017 [acesso em $2018 \mathrm{dez}$ 27];43(119-20):143-53. Disponível em: http://periodicos.unisantos.br/leopoldianum/article/view/741/623

3. Moraes MVG. Doenças ocupacionais: agentes: físico, químico, biológico, ergonômico. $2^{\underline{a}}$ ed. São Paulo: Érica; 2014. 
4. Gonçalves RMV, Schneider KS. Estratégias de enfrentamento da Síndrome de Burnout na enfermagem. Cad Saúde Desenvolv [Internet]. 2016 [acesso em 2017 ago 29];8(5):50-63. Disponível em: https://www.uninter.com/cadernosuninter/index.php/saude-e-desenvolvimento/article/view/435

5. Avelino DC, Silva PMC, Costa LFP, Azevedo EB, Saraiva AM, Ferreira Filha MO. Trabalho de enfermagem no centro de atenção psicossocial: estresse e estratégias de coping. Rev Enferm UFSM [Internet]. 2014 [acesso em 2017 nov 27];4(4):718-26. Disponível em:

https://periodicos.ufsm.br/reufsm/article/view/14163/pdf

6. Dias GC, Furegato ARF. Impacto do trabalho e satisfação da equipe multiprofissional de um hospital psiquiátrico. Rev Enferm UERJ [Internet]. 2016 [acesso em 2019 abr 16];24(1):e8164. Disponível em: http://www.facenf.uerj.br/v24n1/v24n1a14.pdf

7. Sousa KHJF, Gonçalves TS, Silva MB, Soares ECF, Nogueira MLF, Zeitoune RCG. Riscos de adoecimento no trabalho da equipe de enfermagem em um hospital psiquiátrico. Rev Latinoam Enferm [Internet]. 2018 [acesso em 2019 abr 17];26:e3032. Disponível em: http://www.scielo.br/pdf/rlae/v26/pt_0104-1169-rlae-26-e3032.pdf

8. Gil-Monte PR, Carlotto MS, Câmara SG. Validação da versão brasileira do "cuestionario para la evaluación del Síndrome de Quemarse por el Trabajo” em professores. Rev Saúde Pública [Internet]. 2010 [acesso em 2019 abr 17];44(1):140-7. Disponível em: http://www.scielo.br/pdf/rsp/v44n1/15.pdf

9. Costa VS. Desenvolvimento e avaliação de impacto de intervenção preventiva para Síndrome de Burnout em cuidadores residentes [dissertação]. Porto Alegre: Pontifícia Universidade Católica do Rio Grande do Sul; 2015. 69 p.

10. Barros EO. Síndrome de Burnout em enfermeiros que atuam na Estratégia de Saúde da Família [dissertação]. João Pessoa: Universidade Federal da Paraíba; 2016. 80 p.

11. Rodriguez SYS. Burnout em psicólogos: prevalência e fatores associados [tese]. Porto Alegre: Pontifícia Universidade Católica do Rio Grande do Sul; 2015.

12. Corrêa JS. Bem-estar no trabalho e Síndrome de Burnout: análise de faces opostas no cotidiano de servidores penitenciários gaúchos [dissertação]. Santa Maria: Universidade Federal de Santa Maria; 2018. $153 \mathrm{p}$.

13. Gil-Monte PR. Manual do CESQT- Cuestionario para la Evaluación del Síndrome de Quemarse por el Trabajo. Madrid (ES): TEA Ediciones; 2011.

14. Vieira GLC. Satisfação e sobrecarga de trabalho entre técnicos de enfermagem de hospitais psiquiátricos. Rev Port Enferm Saúde Mental [Internet]. 2017 [acesso em 2019 abr 18];(17):43-9. Disponível em: http://www.scielo.mec.pt/pdf/rpesm/n17/n17a06.pdf 
15. Zgiet J. Reforma psiquiátrica e os trabalhadores da saúde mental: a quem interessa mudar? Saúde Debate [Internet]. 2013 [acesso em jul 11];37(97):313-23. Disponível em: https://www.scielosp.org/pdf/sdeb/2013.v37n97/313-323/pt

16. Spiendler Rodriguez SY, Carlotto MS. Prevalência e Fatores Associados à Síndrome de Burnout em Psicólogos. Cienc Trab [Internet]. 2014 [acesso em 2018 jan 05];16(51):170-6. Disponível em: https://scielo.conicyt.cl/pdf/cyt/v16n51/art08.pdf

17. Dutra LB, Aerts D, Alves GG, Câmara SG. A Síndrome de Burnout em docentes do ensino superior de instituições privadas de Santarém, PA. Tempus (Brasília) [Internet]. 2016 [acesso em abr 12];10(3):115-36. Disponível em: http://www.tempusactas.unb.br/index.php/tempus/article/view/1872/1697

18. Zanatta AB, Lucca SR. Prevalência da Síndrome de Burnout em profissionais da saúde de um hospital oncohematológico infantil. Rev Esc Enferm USP [Internet]. 2015 [acesso em 2019 abr 13];49(2):253-8. Disponível em: http://www.scielo.br/pdf/reeusp/v49n2/pt_0080-6234-reeusp-49-02-0253.pdf

19. França SPS, Martino MMF, Aniceto EVS, Silva LL. Preditores da Síndrome de Burnout em enfermeiros de serviços de urgência pré-hospitalar. Acta Paul Enferm [Internet]. 2012 [acesso em 2019 abr 13];25(1):68-73. Disponível em: http://www.scielo.br/pdf/ape/v25n1/v25n1a12.pdf

20. Pantoja FGB, Silva MVS, Andrade MA, Santos AAS. Avaliação do Burnout em trabalhadores de um hospital universitário do município de Belém (PA). Saúde Debate [Internet]. 2017 [acesso em 2019 abr 13];41(Esp 2):200-14. Disponível em:

https://www.scielosp.org/article/ssm/content/raw/?resource_ssm_path=/media/assets/sdeb/v41nspe2/01031104-sdeb-41-spe2-0200.pdf

21. Oliveira RF, Lima GG, Vilela GS. Incidência da Síndrome de Burnout nos profissionais de enfermagem: uma revisão integrativa. Rev Enferm Cent-Oeste Min [Internet]. 2017 [acesso em 2019 abr 18];7:e1383. Disponível em: http://www.seer.ufsj.edu.br/index.php/recom/article/view/1383

22. Silva AP, Carvalho ES, Cardim A. Trabalho noturno na vida dos enfermeiros. Rev Enferm Contemp [Internet]. 2017 [acesso em 2018 jan 05];6(2):177-85. Disponível em:

https://www5.bahiana.edu.br/index.php/enfermagem/article/view/1292/1078

23. Vidotti V, Ribeiro RP, Galdino MJQ, Martins JT. Síndrome de Burnout e o trabalho em turnos na equipe de enfermagem. . Rev Latinoam Enferm [Internet]. 2018 [acesso em 2019 abr 17];26:e3022. Disponível em: http://www.revistas.usp.br/rlae/article/view/154239/150473

24. Fernandes LS, Nitsche MJT, Godoy I. Sindrome de Burnout em profissionais de enfermagem de uma unidade de terapia intensiva. Rev Pesqui Cuid Fundam [Internet]. 2017 [acesso em 2018 jan 05]; 9(2):551-7. Disponível em: http://www.seer.unirio.br/index.php/cuidadofundamental/article/view/4199/pdf_1

25. Alves S, dos-Santos R, Oliveira R, Yamaguchi M. Serviços de saúde mental: percepção da enfermagem em relação à sobrecarga e condições de trabalho. Rev Pesqui Cuid Fundam [Internet]. 2018 [acesso em 
2019 abr 13];10(1):25-9. Disponível em:

http://www.seer.unirio.br/index.php/cuidadofundamental/article/view/5929

\section{Autor correspondente}

Ana Rebeca Soares de Medeiros

E-mail: becasmedeiros@hotmail.com

Endereço: Av. José Caetano, 296, Macapá, Amapá.

CEP: 68.903-605.

\section{Contribuições de Autoria}

1 - Ana Rebeca Soares de Medeiros

Concepção e projeto; coleta de dados; análise e interpretação dos dados; redação do artigo e revisão crítica relevante do conteúdo intelectual.

2 - Carla Braz Evangelista

Concepção e projeto; análise e interpretação dos dados; redação do artigo e revisão crítica relevante do conteúdo intelectual; e aprovação final da versão a ser publicada.

3 - Ronny Anderson de Oliveira Cruz

Análise e interpretação dos dados; redação do artigo; revisão crítica relevante do conteúdo intelectual; e aprovação final da versão a ser publicada.

4 - Ericka Holmes Amorim

Análise e interpretação dos dados; redação do artigo; revisão crítica relevante do conteúdo intelectual.

Autor 5 - José Madson Medeiros Souza

Análise e interpretação dos dados; redação do artigo; revisão crítica relevante do conteúdo intelectual.

\section{Como citar este artigo}

Medeiros ARS, Evangelista CB, Cruz RAO, Amorim EH, Souza JMM. O burnout em profissionais de enfermagem que atuam em um complexo psiquiátrico. Rev. Enferm. UFSM. 2019 [Acesso em: Anos Mês Dia];vol e2: P1-P16. DOI:https://doi.org/10.5902/2179769236205 